\title{
Clinical experience with the Wiktor stent in native coronary arteries and coronary bypass grafts
}

\author{
Sudhir Vaishnav, Sohail Aziz, Clive Layton
}

\begin{abstract}
Objectives-To evaluate the results of implantation of Wiktor tantalum wire coronary stents in stenosed or occluded coronary vessels or in saphenous vein bypass grafts.
\end{abstract}

Design-A retrospective analysis of clinical and angiographic data from patients treated with tantalum wire stents implanted by one operator at two centres.

Patients-52 patients undergoing conventional balloon angioplasty had 67 lesions treated by stents after acute or threatened closure of the target vessel, or because the lesions concerned were considered to be at particularly high risk of becoming restenosed, or because the result of primary angioplasty was inadequate.

Results-65 of the 67 lesions were successfully stented although in two cases the first attempt failed and a second stent was then implanted successfully. There were no cases of stent occlusion and no myocardial infarctions in hospital or in the follow up period of 1-20 months. Eight patients had haemorrhagic complications that were minor in 4 . One patient later had coronary bypass surgery after failure to stent a lesion. Angiographic follow up at a mean of 6 months after stenting showed restenosis associated with 4 of 47 stents studied. All patients with chest pain had had repeat angiography, and $84 \%$ of those without symptoms also agreed to reinvestigation after about 6 months.

Conclusions-The Wiktor tantalum wire stent is an effective means of treating acute complications during angioplasty and seems to offer hope of a significant reduction in the rate of late restenosis in both native coronary vessels and saphenous vein bypass grafts. A prospective comparison of balloon angioplasty and stenting is needed.

(Br Heart f 1994;72:288-293)

Transluminal coronary angioplasty is now established as a routine treatment for patients with suitable coronary obstructive lesions and appropriate clinical indications for intervention. The technique has also been used successfully in patients with stenosis or recent occlusion in coronary bypass grafts. Several problems remain to be solved, however, including the optimal ways of managing acute complications of angioplasty such as actual or impending occlusion, dissection, or inadequate result of dilatation. A high rate of restenosis is also a common feature in follow up angiographic studies and this seems to be particularly troublesome in bypass grafts. Attempts to deal with these problems have included the use of intracoronary stents of which a number of different designs are available. Initial experience with the Wiktor tantalum wire stent confirmed its value as a bail out manoeuvre for acute angioplasty complications, ${ }^{12}$ but its use was associated with subacute stent occlusion and a rate of restenosis comparable with that seen after balloon angioplasty. We report here our experience of the use of Wiktor stents (Medtronic) in a series of patients treated by a single operator with a standardised protocol for treatment before, during, and after the implantation procedure.

\section{Patients and methods}

PATIENT DETAILS

Sixty nine Wiktor coronary stents have been used to treat 67 lesions in 52 patients comprising five women and 47 men with an age range of 38 to 80 years. All patients had been assessed as being suitable for treatment by elective transluminal angioplasty on the basis of clinical and angiographic data with the exception of one case where angioplasty and stent implantation were performed in the course of terminal management of intractable unstable angina with cardiogenic shock. The indications for angioplasty were stable angina in 39 cases, unstable angina in eight cases, and angina after infarction in five cases. The table shows indications for stenting and the vessels treated. In cases with a poor angiographic result or flow limiting vessel dissection stenting was used only after repeated balloon inflations with the largest acceptable size of balloon for the vessel concerned.

Single vessel disease was present in 18 patients and multivessel disease in the remaining 34 . In 13 cases other disease was 
Table Indications for stenting and sites

\begin{tabular}{llclclc}
\hline Indication & $L M S$ & $L A D$ & $L C X$ & $R C A$ & $S V G$ & Total \\
\hline Elective & 1 & 1 & 1 & 3 & 28 & 34 \\
Restenosis & & 8 & 4 & 5 & & 17 \\
Poor result & & 6 & 1 & 1 & & 8 \\
Dissection & 1 & 17 & 1 & 5 & 28 & 67 \\
Total & 1 & 7 & 14 & 28 & 67
\end{tabular}

LMS, left main stem; LAD, anterior descending; LCX, circumflex; RCA, right coronary; SVG, saphenous vein graft. the adjacent vessel. The balloon was deflated and allowed to fully wrap before being gently advanced to ensure that it moved freely within the stent before removal. In one case the stent did not expand fully and a second inflation of the balloon was required.

The patients were returned to the progressive care unit where electrocardiographic and pressure monitoring were continued and the femoral sheaths left in situ. Heparin was continued at a dose sufficient to maintain the activated clotting time between 200 and 250 seconds. Intravenous glyceryl trinitrate was given at an infusion rate of $1 \mathrm{mg} /$ hour for 18 hours and oral drugs continued as before. A further dose of warfarin $(10 \mathrm{mg})$ was given on the evening of the procedure. Cardiac enzymes were measured six hours after the procedure and on the next morning. A 12 lead electrocardiogram was recorded immediately after the procedure, daily thereafter, and at any time the patients complained of chest pain. A full blood count and international normalised ratio (INR) were measured on the morning after the procedure and the dose of warfarin adjusted as necessary. At that time the heparin infusion was temporarily stopped until the activated clotting time fell below 200 seconds when the femoral sheaths would be removed. After haemostasis had been achieved heparin was restarted, if necessary with a bolus dose, to restore the activated clotting time to a level in the range 200 to 250 seconds. When the INR had exceeded 2.5 heparin was stopped and the INR measured again four hours later. If $<2$ at that stage heparin would be restarted for a further 24 hours, otherwise the patient would be maintained on warfarin alone. Aspirin, a calcium antagonist, and an oral long acting nitrate were also given throughout.

Patients were discharged from hospital once heparin had been discontinued and the INR was confirmed to be $\geqslant 2 \cdot 2$, with the intention of maintaining the INR subsequently in the range 2.5 to 4 . Aspirin and a calcium antagonist were also continued after discharge from hospital but nitrates were not given at that stage. The calcium antagonist was normally withdrawn one month after the procedure unless needed for treatment of hypertension or to control residual angina. Warfarin was withdrawn after three months and low dose aspirin continued indefinitely if tolerated. All patients were offered follow up angiography about six months after the procedure or earlier if they complained of chest pain.

Angiograms were analysed by an independent observer by direct measurement of vessel diameter at the most severe point of narrowing after manual definition of the vessel edges on cineangiograms. This was expressed as a percentage of the size of the nearest normal adjacent segment of the same vessel measured in the radiographic projection showing the most severe degree of stenosis. Restenosis was similarly measured and defined as a reduction in luminal diameter of $\geqslant 50 \%$. be $0.5 \mathrm{~mm}$ larger than the balloon previousl used to achieve a stent diameter at least equal to or marginally greater than the diameter of 


\section{Results}

Sixty nine stents were used but one of these was positioned in a branch of the femoral artery after unsuccessful stenting of the coronary lesion at the ostium of the right coronary artery. A second stent was successfully implanted in the lesion. A further stent was positioned in a circumflex artery but not at the site of the target lesion, which could not be successfully crossed despite satisfactory dilatation. Two other stents were removed by a basket extraction device (KeyMed) that became available to us only after the first two cases in which the stents were left in the patients. In these cases the guide wire was retained in the coronary artery and the balloon was inflated to a pressure of $0.5 \mathrm{bar}$ in the ascending aorta to trap the stent on the balloon. The balloon and guiding catheter were then withdrawn until the guiding catheter had been fully removed and the stent was at the mouth of the sheath in the external iliac artery. It was then snared and removed. One of the two cases managed in this way was successfully stented with a second stent. The overall success rate of the stenting was therefore 65/67 lesions representing $97 \%$. None of the patients needed urgent or emergency coronary bypass surgery and no patient had a myocardial infarct as judged by serial electrocardiograms and enzyme analysis.

The mean (range) nominal size of stent used was 3.74 (3.0 to 4.5$) \mathrm{mm}$. Because of the compliance of the balloon and the routine use of a pressure of 8 bar to expand the stent the actual stent size is expected to be about $5 \%$ greater than the nominal diameter. The size of stent used in coronary bypass grafts was greater than that used in native coronary arteries (4.32 v3.63 mm). The mean (range) nominal size of balloon used for dilatation of the lesions was $3.37(2.5$ to 4.0$) \mathrm{mm}$.

Of the two patients in whom stenting failed one elected to have coronary bypass surgery two months later and the second patient was treated conservatively. One patient died 36 hours after the procedure; this patient had previously had coronary bypass surgery and all except one graft had occluded. This final graft was critically stenosed and the patient was transferred from another hospital with severe unstable angina and left ventricular failure unresponsive to conventional treatment. The remaining bypass graft was dilated and stented in the hope of stabilising the situation and the procedure itself was uneventful. Subsequently the patient experienced no further chest pain but the cardiac failure proved intractable and the patient died as a result of progressive irreversible loss of cardiac output. The remaining patients were all discharged from hospital uneventfully and there were no cases of stent occlusion.

In one case with an ostial stenosis of a coronary bypass graft the stent was successfully positioned but the proximal loop of the stent was damaged by the guiding catheter while a check angiogram was performed after the stent balloon catheter had been withdrawn. The tip of the guiding catheter caught on the end of the stent and when it was withdrawn pulled the proximal loop straight leaving a short length of wire protruding into the aorta. No complication was associated with this but the technique for ostial lesions was subsequently modified to avoid a recurrence of this complication.

Distal embolisation of atheromatous material or thrombus occurred during the procedure in five cases. Two of these patients were treated with streptokinase and the target vessel remained patent in all cases.

Other acute complications were mainly related to bleeding from the femoral puncture site in four cases. In one case this needed transfusion of two units of blood. This patient had been treated with streptokinase for coronary thrombosis before stenting. In all cases bleeding was controlled by local compression and reduction of anticoagulation with removal of the femoral sheaths. One patient was treated with protamine to lower the activated clotting time to facilitate haemostasis. No cases needed urgent surgical repair but two patients developed false aneurysms of the femoral artery four and seven weeks after the procedure. Both were repaired uneventfully. Two patients experienced late gastrointestinal haemorrhagic complications needing transfusion. In one case the site of bleeding was not found and bleeding stopped after discontinuing warfarin nine weeks after stenting. In the second case the patient, contrary to instructions, had continued to take warfarin until 25 weeks after stenting when a major bleed occurred from a duodenal ulcer. This responded to transfusion and conservative treatment with withdrawal of warfarin. Haematuria, iron deficiency anaemia, and gum bleeding each occurred in one case.

One patient developed acute renal failure after stenting associated with septicaemia. This patient had been treated with intravenous drugs through central venous lines for unstable angina for several days before the procedure. She required haemodialysis for two weeks before making a full recovery.

Angiographic follow up at one to seven (mean 6.0) months after stenting is available for 37 patients with 47 stents implanted. These include 20 vein graft stents in 15 patients and 27 stents in native vessels in 22 patients. All patients who complained of chest pain underwent angiography. Five patients who were offered follow up angiograms declined as they were free from symptoms. Restenosis was found in four out of 47 stents, an angiographic rate of restenosis of $8.5 \%$ expressed as the percentage of those stents for which angiographic data are available. Two of these patients complained of angina. Two of the stents which showed restenosis were in native vessels and two were in bypass grafts.

Four patients subsequently had a repeat angioplasty for recurrent angina. In one of these the stent stenosis was redilated and in the rest other lesions elsewhere in the coronary circulation were treated. A further 
patient is awaiting repeat dilatation for stent stenosis.

\section{Discussion}

Coronary stents have been developed in response to the need to resolve several problems related to coronary angioplasty, ${ }^{3}$ in particular, management of the acute complications of dissection with severe luminal encroachment, acute vessel closure, and impending closure. These have traditionally been dealt with by the use of perfusion balloons often followed by urgent referral for surgery. ${ }^{4}$ With the use of stents it is hoped to avoid the need for such action. Our experience with the Wiktor tantalum wire stent suggests that the use of this device can considerably reduce the need for perfusion balloons and can be accomplished at low risk with excellent long-term results. We have used no perfusion balloons during the period covered by this report.

The main disadvantage currently associated with coronary angioplasty is the high rate of restenosis, ${ }^{56}$ and this problem has been particularly noted when treating stenoses in coronary bypass grafts where rates of restenosis of $>60 \%$ have been reported. ${ }^{78} \mathrm{We}$ have used the Wiktor stent electively in treating bypass graft stenosis with excellent long-term results similar to those seen in native coronary arteries.

The technique of stent implantation needed modification in patients with ostial lesions after the damage caused to an ostial stent by the guiding catheter. In subsequent cases the stent was passed to a point several centimetres beyond the ostial lesion and the guiding catheter was then withdrawn into the aorta. The stent was then pulled back to the correct position and was placed without support from the guiding catheter. The guiding catheter was not then reinserted into the coronary artery but the position of the stent was checked by injection of contrast medium into the aortic root. No further cases of stent damage were seen when this technique was used.

One of the striking features noted in our series was the ability of the Wiktor stent to adapt to the shape of the vessel, in particular where the lesion occurred near a sharp bend in the vessel. Although during positioning of the stent the vessel would straighten, after removal of the balloon the stent showed an exceptional capacity to adopt to the shape of the vessel even when complex bends were involved. Furthermore the design of the stent ensured excellent perfusion through side branches whose origins were covered by the stent. This was particularly well shown in the case where a left main stem lesion was treated by a stent with the origin of the main circumflex artery completely covered by the stent. In common with other types of stent the balloon with the stent premounted is fairly rigid and we have therefore been careful to avoid trying to position the stent in vessels where access to the lesion was likely to have been very diffi- cult. For that reason the number of circumflex arteries treated by stenting is small in comparison with the other vessels and it is clear from our experience that successful positioning of a stent in the distal circumflex system does pose additional technical problems of access to the lesion. Once that has been achieved the conformability of the positioned and unmounted stent is excellent. Improved design of the delivery system of the balloon in the future may help to overcome this difficulty.

The Wiktor stent seems to have considerable advantages over the Palmaz-Schatz stent in two main respects. The Wiktor stent is easily seen on fluoroscopy after placement enabling quick assessment of the effectiveness of the balloon at expanding the stent fully. This is in contrast with the Palmaz-Schatz stent which is difficult to see and confirmation of an accurate position can be a problem. The design of the tantalum wire Wiktor stent also allows the positioning of the stent across major side branches without apparent interference in flow in those vessels as exemplified by the patient with the stent in the left main artery across the origin of the main circumflex artery.

Our results both in native vessels and bypass grafts are at odds with those reported by others who used stents of various designs. ${ }^{9-12}$ We have compared our technique with that of other workers to try and identify possible areas of technical difference that may help to account for these different results. One striking feature is the total lack of acute occlusion of stents in our series. We ascribe this to a number of possible factors. Firstly we have endeavoured to use the largest possible stent size compatible with the size of vessel being treated and at the same time have avoided overdistension. This is reflected by the large mean nominal stent diameter recorded. Secondly, we have developed and applied a rigid management protocol for control of anticoagulation during and after the procedure, which we believe helps to minimise the risk of wildly fluctuating levels of anticoagulation. This may have relevance to both the acute rate of stent occlusion and also the rate of late restenosis, the seeds of which may be sown in the first few days after stent implantation and even during the procedure itself. Previous studies have shown that stent occlusion is associated with the use of small stents and in particular those with a diameter of $<3.0 \mathrm{~mm} .{ }^{11}$ On the other hand restenosis after conventional balloon angioplasty seems to occur more commonly when the target lesion is overdilated and it has been recommended that deliberate suboptimal dilatation may be helpful. ${ }^{13}$ This does not seem to apply to stent implantation. We found no relation between stent size and restenosis.

Our protocol depends heavily on the skill and motivation of the nursing staff who are responsible for monitoring and controlling heparin concentrations and removing the femoral sheaths, but despite the rigidity and demanding nature of the protocol it has 
proved easy to operate and there has been little difficulty in maintaining levels of anticoagulation within a fairly narrow range. Although local bleeding was a common problem this was almost always minor and could always be controlled by local pressure on the femoral artery. Transfusion was needed only in a patient who had also received thrombolytic treatment with streptokinase for an acutely occluded right coronary artery associated with continuing unstable angina before stent implantation.

In the early part of the series both dipyridamole and dextran 40 were used but were abandoned at an early stage. There seemed to be no advantage to their use and we, in common with others, have doubts about their value. ${ }^{14}$ Dipyridamole was associated with a high incidence of patient intolerance; many patients discontinued the drug themselves after a few weeks of treatment. Dextran 40 did not seem to offer any benefit as no cases of stent occlusion occurred either with or without dextran. When dextran was used, however, problems were encountered as a result of the considerable haemodilution effect that both caused or exacerbated cardiac failure in patients with poor left ventricular function, and also caused low haemoglobin concentrations in the first 24 hours after implantation of the stent. In some cases this raised the possibility of silent internal haemorrhage and resulted in unnecessary investigations.

False aneurysm of the femoral artery occurred in two patients and one of these was an abrupt event that developed over a period of 24 hours seven weeks after the procedure. Both of these aneurysms were successfully repaired without complications but necessitated a further admission to hospital and anaesthetic. Factors known to cause increased local haemorrhage and vascular complications include the size of catheters and use of anticoagulation or thrombolysis. ${ }^{15}$ In this series size 8 French guiding catheters were used throughout. There were no obvious differences in anticoagulant control in these cases compared with the other patients and although one of the patients was obese the other was not. In neither case had prolonged bleeding occurred when the femoral sheaths were removed, nor had any difficulty been encountered either in insertion or removal of the sheaths. The policy of keeping the sheaths in place overnight may contribute to this problem as we have not experienced formation of a femoral pseudoaneurysm in patients undergoing conventional angioplasty, in whom the sheaths are removed a few hours after the procedure. Although the sheaths were kept in situ for 18 hours after stenting none of the patients had repeat angiography during that period and we are therefore reviewing the need to keep the sheaths in place for this prolonged period. We believe that the incidence of pseudoaneurysm may be reduced if the sheaths are removed earlier but this must be set against the need to temporarily reduce the level of anticoagulation soon after stent implantation if an earlier removal policy is to be adopted. This may have adverse effects in terms of early stent occlusion. We did not use any other devices to control local bleeding after removal of the sheaths as our past experience of collagen plugs and pressure devices has been unsatisfactory.

Several published studies reported the incidence of early stent occlusion and late restenosis with various types of coronary stent. All of these studies have shown considerable occlusion rates and most also show a rate of restenosis comparable with that expected after conventional coronary angioplasty. The unexpectedly good results in our series may reflect an underlying selection bias towards low risk cases although analysis of the type of patients and lesions treated does not seem to support this view and the results were not different in patients previously treated by angioplasty and in whom restenosis was the indication for the current procedure. Alternatively some aspects of the technique of implantation and subsequent medical management combined with the structure and design of the stent itself may be contributing to a genuine reduction in the rates of early occlusion and late restenosis.

The results obtained in this group of patients who were selected on purely clinical grounds suggest that the use of Wiktor tantalum wire stents with a carefully defined protocol for managing the anticoagulant regime subsequently may have a substantial effect in reducing the number of cases needing emergency surgery for complications of angioplasty but also could help to reduce the otherwise intractable rate of restenosis seen after balloon dilatation. The rate of restenosis seen in this study suggests an impressive potential reduction compared with the expected rate after balloon dilatation, particularly in the patients with saphenous vein bypass grafts. The patients, however, were not randomly allocated and no direct comparison can therefore be validly made. This highlights the need for a randomised prospective study to compare angioplasty with stenting as the primary management for suitable coronary lesions both in native vessels and bypass grafts.

Copies of the management protocol used in this series of patients may be obtained from the authors on request.

1 Buchwald A, Unterberg C, Werner G, Voth E, Kreuger $H$, Wiegand $V$. Initial clinical results with the Wiktor stent: a new balloon-expandable coronary stent. Clin Cardiol 1991;14:374-9.

2 de Jaegere PP, Serruys PW, Bertrand M, et al. Wiktor stent implantation in patients with restenosis following balloon angioplasty of a native coronary artery. $\mathrm{Am}$ Cardiol 1992;69:598-602.

3 Sigwart U, Puel J, Mirkovitch V, Joffre F, Kappenberger $\mathrm{L}$. Intravascular stents to prevent occlusion and resteno1987;316:701-6.

4 Sundram P, Harvey JR, Johnson RG, Schwartz MJ, Baim DS. Benefit of the perfusion catheter for emergency coronary artery grafting after failed percutaneous coro-

5 Popma JJ, Topol EJ. Factors influencing restenosis after coronary angioplasty. Am $₹$ Med 1990;88:1N-24N. 
6 Califf RM, Fortin DF, Frid DJ, et al. Restenosis after coronary angioplasty: an overview. $\mathfrak{f} \mathrm{Am}$ Coll Cardiol 1991;17:2B-13B.

7 Pepine CJ, Hirshfield JW, MacDonald RG, et al. A controlled trial of corticosteroids to prevent restenosis after coronary angioplasty. Circulation 1990;81: 1753-61.

8 Platko WP, Hollman J, Whitlow PL, Franco I. Percutaneous transluminal angioplasty of saphenous vein graft stenosis: long-term follow-up. $f \mathrm{Am} \mathrm{Coll}$ vein graft stenosis: lon

9 Kiemeneij F, Laarman GJ, van der Wieken $R$ Suwarganda J. Emergency coronary stenting with the Palmaz-Schatz stent for failed transluminal coronary angioplasty: results of a learning phase. Am Heart $f$ angioplasty: result

10 Haude $M$, Erbel $R$, Issa $H$, et al. Subacute thrombotic complications after intracoronary implantation of Palmaz-Schatz stents. Am Heart $\mathcal{f}$ 1993;126:15-22.
11 Carrozza JP, Kuntz RE, Levine MJ, et al. Angiographic and clinical outcome of intracoronary stenting: immediate and long-term results from a large single-center experience. F Am Coll Cardiol 1992;20:328-37.

12 Serruys PW, Strauss BH, Beatt KJ, et al. Angiographic follow-up after placement of a self-expanding coronaryartery stent. N Engl ₹ Med 1991;324:13-7.

13 Serruys PW, Foley DP, de Feyter PJ. Restenosis after coronary angioplasty: a proposal of new comparative coronary angioplasty: a proposal of new comparative approaches based

14 Simon R, Hermann G, Zahorsky R, Wille B, Nellessen U, Ould G. Coronary stenting: are dextran and dipyridamole really

15 Muller DWM, Shamir KJ, Ellis SG, Topol EJ. Peripheral vascular complications after conventional and complex percutaneous coronary interventional procedures. $A m$ Cardiol 1992;69:63-8.

\section{IMAGES IN CARDIOLOGY}

\section{Near miss paradoxical embolism}

Swelling of the right calf developed in a 70 year old woman after a 5 hour coach trip. She presented 2 weeks later with breathlessness, hypoxia, and a normal chest $x$ ray. The ventilation-perfusion scan confirmed pulmonary embolism; despite treatment with intravenous heparin she became gradually more breathless. Echocardiography showed a large tubular mass attached to the interatrial septum and extending across the right atrium and ventricle. At thoracotomy there was a large thrombus (figure) with its proximal end crossing a patent foramen ovale and its distal end free in the right ventricular outflow tract. This was removed without complication.

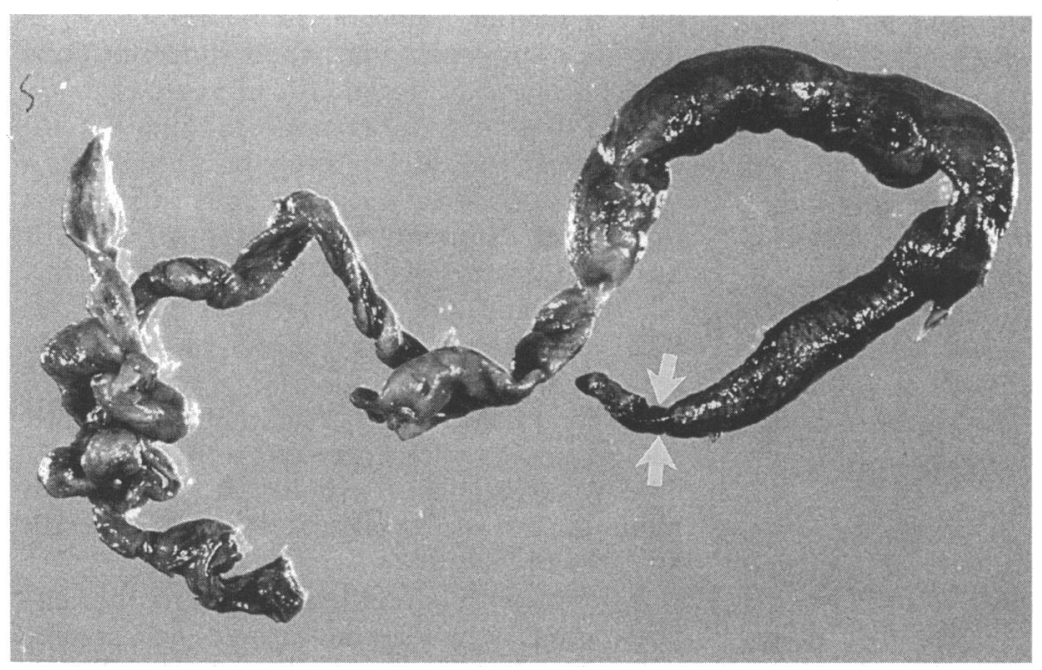

Thrombus removed from the right heart at thoracotomy. The proximal end had crossed a patent foramen ovale into the left atrium as far as the level of the arrows.
The evidence that a patent foramen ovale can cause an embolic stroke remains circumstantial. A patent foramen ovale was detected in around $15 \%$ of patients after cerebral infarction, ${ }^{12}$ but it was also detected in a similar proportion of controls. ${ }^{3}$ The mechanism for stroke requires that a thrombus should pass close to the patent foramen ovale at the same time as there is a reversal of the normal left to right atrial pressure difference. This apparently unlikely sequence of events can occur during pulmonary embolism when even a large thrombus may catch in a patent foramen ovale as is illustrated by this case.

There are few studies of the leg and intraabdominal veins after stroke. ${ }^{4} \mathrm{We}$ believe that research of this type is the next step in assessing the clinical significance of patent foramen ovale in patients with cerebral infarction.

A PRAKASH

J CHAMBERS

P HOLT

1 Pearson AC, Labovitz AJ, Tateneni S, Gomez CR. Superiority of transesophageal echocardiography in detecting cardiac source of embolism in patients with cerebral ischaemia of uncertain etiology. $\mathcal{f} \mathrm{Am}$ Coll Cardiol 1991;17:66-72.

2 de Belder MA, Lovat LB, Tourika L, Leech G, Camm AJ. Limitations of transoesophageal echocardiography in patients with focal cerebral ischaemic events. Br Heart 1992;67:367-77.

3 Vandenbogaerde $\mathrm{J}$, de Bleeker $\mathrm{J}$, Decoo $\mathrm{D}$. Transesophageal echocardiography in patients suspected of a cardiac source of emboli. Eur Heart $\mathcal{f}$ 1992;13:88-94.

4 Stöllberger C, Slany J, Schuster I, Leitner H, Winkle W-B, Karnik R. The prevalence of deep venous throm bosis in patients with suspected paradoxical embolism. bosis in patients with suspected 